\title{
Intragenic Deletions May Involve Enhancer Sequences and Alter CNTNAP2 Expression
}

With the development of ever higher resolution arrayCGH platforms, detection of copy number variations (CNVs) as small as a single exon came within reach [Boone et al., 2010]. Meanwhile, whole-genome sequencing techniques brought the limit of detection down to the nucleotide level. Several molecular mechanisms can be envisaged to explain a possible contribution of a deletion or duplication of one or several genes or of a single exon to the phenotype(s) of a patient. First, phenotypic effects of deletions or duplications of one or a few exons of one allele may involve alterations in the protein domain(s) encoded by the exon(s) [Poot and Haaf, 2015; Poot, 2017; Veitia et al., 2018]. Second, if an intragenic deletion involves a transcription factor binding site, an imbalance between the levels of transcription factor protein molecules and their targets may result [Poot and Haaf, 2015; Poot, 2017]. Third, nonprotein-coding sequence elements, such as long noncoding RNAs, micro RNAs, antisense RNAs, etc., may be lost or duplicated. This may provoke an imbalance between the levels of mRNA and antisense RNA molecules [Poot and Kas, 2013]. A recent case of a patient with an inherited $289.3-\mathrm{kb}$ deletion of CNTNAP2 including exons 14-15 does not convincingly fit into any of the 3 models outlined above and prompts to look for other possible explanations [Lee et al., 2015; Flaherty et al., 2017].

Exon 15 is the first exon encoding the third Laminin G domain of CNTNAP2 [Poot, 2017]. If the allele with the deletion of exons 14-15 would be expressed, a CASPR2 protein lacking this domain would result. Such a CASPR2 protein would then compete with the full-length CASPR2

\section{KARGER}

(C) 2018 S. Karger AG, Basel

E-Mail karger@karger.com

www.karger.com/msy protein for its putative protein partners on another cell membrane and thus exert a dominant negative action. Since CNTNAP2 is only poorly expressed in cultured fibroblasts, another cell type is needed for functional studies. Human induced pluripotent stem cells (hiPSCs) derived from a daughter who met DSM-IV criteria for a schizoaffective disorder and her unaffected father (both carrying the 289.3-kb deletion) were used to investigate CNTNAP2 expression patterns [Lee et al., 2015]. From the hiPSC, neural progenitor cells (NPCs) were derived, which were induced to become glutametergic neurons with Ngn2. In NPCs of the daughter and the unaffected father, expression of the deleted exons 14-15 was lower than in the healthy mother. In contrast, both carriers showed increased expression of exons 23-24 of CNTNAP2. In hiPSC-derived forebrain neurons, a significantly increased CNTNAP2 expression of exons 2-3 and exons 23-24 was observed in the proband only, suggesting that the exon 14-15 deletion leads to increased expression of this allele, which is in agreement with the clinical phenotype. In Ngn2-induced neurons, which had reached electrophysiological maturity, again significantly decreased expression of the deleted exons $14-15$ was detected in the affected daughter only. These findings agree with a study of allele-biased expression in hiPSC-derived neurons, which indicated allele-biased expression of putative schizophrenia and autism-associated genes, such as CNTNAP2 [Lin et al., 2012]. In Ngn2-induced neurons, but not in NPC and astrocytes, overall CNTNAP2 expression was increased in both CNTNAP2 deletion carriers compared with the noncarrier mother [Flaherty et al.,

Martin Poot

Department of Human Genetics, University of Würzburg Biozentrum, Am Hubland DE-97074 Würzburg (Germany)

E-Mail Martin_Poot@hotmail.com 
2017]. Subsequently, differential expression analysis was performed, and the top 500 differentially expressed genes were subjected to enrichment analysis based on Gene Ontology. The most significant subset of genes mapped to Gene Ontology terms relating to DNA binding and central nervous system (CNS) development. Among the gene subset involved in CNS development, genes such as CNTN6 and CNTN4 appeared, which are involved in regulating cell surface interactions during CNS development and are also thought to influence synaptogenesis [Flaherty et al., 2017]. Interestingly, in a cohort of children with autism spectrum disorders, risk alleles of CNTNAP2 and CNTN6 occurred more frequently than expected, assuming random segregation [Poot, 2014]. In an independent cohort of patients with autism spectrum disorders, a single patient inherited mutated alleles of CNTNAP2 and CNTN6 from each parent [Mercati et al., 2017]. Although a mechanistic basis for these observations has not yet been proposed, they implicate differences in exonand allele-specific CNTNAP2 expression in the predisposition to some neurodevelopmental disorders.

A clue towards possible mechanisms for exon- or allele-specific gene expression emerged from research into the function of promoter and enhancer sequences. According to the classic paradigm, active promoters are localized $5^{\prime}$ to the transcription start site (TSS) of a gene. These can be easily discerned since at these sites, the fourth lysine residue of histone $\mathrm{H} 3$ (H3K4) is frequently marked by trimethylation and little by monomethylation [Heintzman et al., 2007, 2009]. In contrast, active enhancers close to TSSs are marked by H3K4 monomethylation, but not trimethylation [Heintzman et al., 2007]. In addition to enhancers close to TSSs, intragenic enhancers, whose chromatin is marked by high levels of $\mathrm{H} 3 \mathrm{~K} 4$ monomethylation and low levels of $\mathrm{H} 3 \mathrm{~K} 4$ trimethylation, have been described [Kim et al., 2010]. These intragenic enhancers may give rise to short bidirectional transcripts, known as eRNAs [Kim et al., 2010]. These eRNAs are not elongated and contain no poly(A) tails [De Santa et al., 2010; Kim et al., 2010]. Thus, promoters and enhancers produce different classes of RNA transcripts: either full-length mRNAs or short eRNAs [Heintzman et al., 2009; Kim et al., 2010]. More recently, a third class of trancripts, multiexonic polyadenylated RNAs, so-called meRNAs were found to be produced by activated intragenic enhancers [Kowalczyk et al., 2012]. These meRNAs appear as isoforms of the full-length gene and add significantly to the already known complexity of the transcriptome.
In addition to the full-length transcript of CNTNAP2, its genomic sequence encompasses several protein-coding and noncoding transcripts, which probably represent meRNAs originating from intragenic enhancers, each functioning as alternative promoters [Lee et al., 2015; Poot, 2015]. Conceivably, transcription of meRNAs from intragenic enhancers would slow transcription of the fulllength mRNA. A recent study showed that this is indeed the case, but not by the envisioned mechanism [Cinghu et al., 2017]. Performing genome-wide RNA Polymerase II (Pol II) chromatin immunoprecipitation sequencing (ChIP-seq) in mouse embryonic stem cells, the authors detected intragenic enrichment of Pol II binding at sites of tens of thousands of base pairs downstream of TSSs. The authors hypothesized that these Pol II binding sites may reflect sites of transcription regulation during productive mRNA elongation. To test this hypothesis, the authors systematically intragenic sites of Pol II enrichment. Thus, they found 1,928 intragenic RNA Pol II sites (IRSs), located at least $1 \mathrm{~kb}$ away from TSSs and transcription end sites of all known and predicted genes. This, by definition, excludes Pol II pausing sites immediately downstream of promoters and near $3^{\prime}$ cleavage or polyadenylation sites. These IRSs exhibit an accessible chromatin architecture, being marked by enhanced DNase I hypersensitivity, high levels of histone modifications $\mathrm{H} 3 \mathrm{~K} 4$ monomethylation, and H3K27 acetylation as associated with active enhancers. These IRSs did not show $\mathrm{H} 3 \mathrm{~K} 4$ trimethylation, which is associated with active promoters [Heintzman et al., 2007; 2009]. Therefore, these IRSs generally represent transcriptionally active intragenic enhancers.

Messenger RNA transcription of intragenic enhancercontaining genes is significantly lower than that of genes without IRSs [Cinghu et al., 2017]. This suggests that transcriptionally active intragenic enhancers somehow attenuate host gene mRNA expression. Thus, it is conceivable that Pol II-dependent transcription from intragenic enhancers on the sense strand may also interfere with Pol II-dependent mRNA transcription of the host gene on the same strand. With CRISPR/Cas9-mediated deletion formation, the authors demonstrate a physiological role for intragenic enhancer-mediated transcription attenuation in cell fate determination during early embryonic development. They also found no evidence of enrichment for R-loops and H3K9 dimethylation at IRSs or RNAi binding factors such as Drosha and Dgcr8 [Chen et al., 2016; Kurimoto et al., 2015: Knuckles et al., 2017]. This suggests that eRNA transcription per se is not sufficient to explain IRSs-dependent transcription interfer- 
ence or attenuation. Therefore, intragenic enhancer transcription alone remains the only plausible explanation for IRSs-mediated transcription attenuation. The authors propose that intragenic enhancers fine-tune transcription of their host gene through transcription interference, thus facilitating a different use of the same regulatory element for disparate functions.

This phenomenon may not be restricted to lineage switching of embryonic stem cells and consequential cell fate decisions during embryonic development, but may also apply to neurodevelopment. For example, for genes with multiple isoforms, such as CNTNAP2, NRXN1, and CNTN5, intragenic deletions may involve losses of intragenic enhancer sites, which in turn engender preferential transcription of the deletion carrying allele [Schaaf et al., 2012; Nava et al., 2014; Lee et al., 2015; Poot, 2017]. In this respect, it may be worthwhile to investigate patient-derived hiPSC neurons by Pol II ChIP-seq [Flaherty et al., 2017].

Martin Poot

\section{References}

Boone PM, Bacino CA, Shaw CA, Eng PA, Hixson $\mathrm{PM}$, et al: Detection of clinically relevant exonic copy-number changes by array CGH. Hum Mutat 31:1326-1342 (2010).

-Chen Y, Pai AA, Herudek J, Lubas M, Meola N, et al: Principles for RNA metabolism and alternative transcription initiation within closely spaced promoters. Nat Genet 48:984-994 (2016).

-Cinghu S, Yang P, Kosak JP, Conway AE, Kumar $\mathrm{D}$, et al: Intragenic enhancers attenuate host gene expression. Mol Cell 68:104-117 (2017).

De Santa F, Barozzi I, Mietton F, Ghisletti S, Polletti S, et al: A large fraction of extragenic RNA pol II transcription sites overlap enhancers. PLoS Biol 8:e1000384 (2010).

-Flaherty E, Deranieh RM, Artimovich E, Lee IS, Siegel AJ, et al: Patient-derived hiPSC neurons with heterozygous CNTNAP2 deletions display altered neuronal gene expression and network activity. NPJ Schizophr 3:35 (2017).

-Heintzman ND, Stuart RK, Hon G, Fu Y, Ching $\mathrm{CW}$, et al: Distinct and predictive chromatin signatures of transcriptional promoters and enhancers in the human genome. Nat Genet 39:311-318 (2007).

-Heintzman ND, Hon GC, Hawkins RD, Kheradpour P, Stark A, et al: Histone modifications at human enhancers reflect global cell-typespecific gene expression. Nature 459:108-112 (2009).
Kim TK, Hemberg M, Gray JM, Costa AM, Bear Dava C, Keren B, Mignot C, Rastetter A, ChantotDM, et al: Widespread transcription at neuronal activity-regulated enhancers. Nature 465: 182-187 (2010).

Knuckles P, Carl SH, Musheev M, Niehrs C, Wenger A, Bühler M: RNA fate determination through cotranscriptional adenosine Poot M: A candidate gene association study furmethylation and microprocessor binding. Nat Struct Mol Biol 24:561-569 (2017).

Kowalczyk MS, Hughes JR, Garrick D, Lynch $\mathrm{MD}$, Sharpe JA, et al: Intragenic enhancers act as alternative promoters. Mol Cell 45:447458 (2012). Bastaraud S, et al: Prospective diagnostic analysis of copy number variants using SNP microarrays in individuals with autism spectrum disorders. Eur J Hum Genet 22:71-78 (2014).

ther corroborates involvement of contactin genes in autism. Mol Syndromol 5:229-235 (2014).

Poot M: Connecting the CNTNAP2 networks with neurodevelopmental disorders. Mol Syndromol 6:7-22 (2015).

Kurimoto K, Yabuta Y, Hayashi K, Ohta H, Kiyo- Poot M: Intragenic CNTNAP2 deletions: a bridge nari $\mathrm{H}$, et al: Quantitative dynamics of chromatin remodeling during germ cell specification from mouse embryonic stem cells. Cell Stem Cell 16:517-532 (2015).

Lee IS, Carvalho CM, Douvaras P, Ho SM, Hartley BJ, et al: Characterization of molecular and cellular phenotypes associated with a heterozygous CNTNAP2 deletion using patient-derived hiPSC neural cells. NPJ Schizophr 1: 15019 (2015).

- Lin M, Hrabovsky A, Pedrosa E, Wang T, Zheng D, Lachman HM: Allele-biased expression in differentiating human neurons: implications for neuropsychiatric disorders. PLoS One 7:e44017 (2012).

Mercati O, Huguet G, Danckaert A, André-Leroux G, Maruani A, et al: CNTN6 mutations are risk factors for abnormal auditory sensory perception in autism spectrum disorders. Mol Psychiatry 22:625-633 (2017). too far? Mol Syndromol 8:118-130 (2017).

Poot M, Haaf T: Mechanisms of origin, phenotypic effects and diagnostic implications of complex chromosome rearrangements. Mol Syndromol 6:110-134 (2015).

Poot M, Kas MJ: Antisense may make sense of 1q44 deletions, seizures, and HNRNPU. Am J Med Genet A 161A:910-912 (2013).

Schaaf CP, Boone PM, Sampath S, Williams C, Bader PI, et al: Phenotypic spectrum and genotype-phenotype correlations of NRXN1 exon deletions. Eur J Hum Genet 20:12401247 (2012).

Veitia RA, Caburet S, Birchler JA: Mechanisms of Mendelian dominance. Clin Genet 93:419428 (2018). 